\title{
Fully inorganic mixed cation lead halide perovskite nanoparticles: a study at the atomic level
}

Tal Binyamin ${ }^{1}$, Laurent Pedesseau ${ }^{2}$, Sergei Remennik ${ }^{1}$, Amal Sawahreh ${ }^{1}$, Jacky Even ${ }^{2}$, and Lioz $\operatorname{Etgar}^{1 *}$

${ }^{1}$ Institute of Chemistry, Casali Center for Applied Chemistry, The Hebrew University of Jerusalem, Edmond J. Safra Campus, Givat Ram, Jerusalem 91904, Israel

${ }^{2}$ University Rennes, INSA Rennes, CNRS, Institut FOTON - UMR 6082, F-35000 Rennes, France

*lioz.etgar@mail.huji.ac.il

\begin{abstract}
Mixed cation perovskites are currently the most efficient perovskite materials used in perovskite solar cells. Mixing two cations inside a perovskite structure results in enhanced flexibility when designing interesting material properties. Moreover, using two inorganic cations in the same perovskite maintains the advantage of fully inorganic structures. A fascinating subject to investigate is therefore the nanoscale synthesis and the properties of such mixed inorganic cation perovskites. In this work we mixed $\mathrm{Rb}$ and $\mathrm{Cs}$ inorganic atoms inside perovskite nanoparticles. We explored down to the atomic resolution different $\mathrm{Rb}$ and $\mathrm{Cs}$ concentrations and performed the chemical mapping of single nanoparticles. At medium concentrations, the $\mathrm{Rb}$ atoms are observed in the core of the particles, whereas the $\mathrm{Cs}$ atoms are located in the shell region, forming core shell structures. However, if there are high concentrations of $\mathrm{Rb}$, a phase separation occurs because bulk perovskite based solely on $\mathrm{Rb}$ cations is not stable at room temperature. Density functional theory calculations support our experimental observations by showing that a stable nanoparticle is formed when the $\mathrm{Rb}$ atoms are located inside the particle and not on the surface. Our work demonstrates the importance of understanding the perovskite structure at the atomic level, leading to the formation of mixed cation bulk perovskites and nanoparticles, and to improved perovskite stability. A new phase of cesium lead bromide $\left(\mathrm{Cs}_{6} \mathrm{~Pb}_{5} \mathrm{Br}_{16}\right)$ related to the $\mathrm{Rb}_{6} \mathrm{~Pb}_{5} \mathrm{Cl}_{16}$ structure is also reported.
\end{abstract}




\section{Introduction}

Metal halide perovskites have attracted much attention in the last decade due to their use as a light harvester in solar cells. In this capacity, these perovskites have achieved remarkable efficiencies in a short time. ${ }^{1}$ The lead halide perovskites are known for their large absorption coefficients, long charge carrier diffusion lengths, low exciton binding energies, tunable band gaps, and the possibility of preparing them from solution. . $^{2,3,4,5,6,7,8}$ This perovskite's chemical formula is $\mathrm{AMX}_{3}$, where $\mathrm{A}$ is a monovalent cation (organic or inorganic), $\mathrm{M}$ is a divalent cation, and $\mathrm{X}$ is a halide. The methylammonium lead iodide is among the most common hybrid organic-inorganic perovskites currently being used. When the organic cation is replaced by an inorganic cation, a fully inorganic perovskite is formed. The main option here, due to size limitations, is the cesium cation $\left(\mathrm{Cs}^{+}\right)$, which can form cesium lead halide perovskite. $\mathrm{CsPbI}_{3}$ is not stable in its bulk form, leading to a yellow non-perovskite phase; however, nanoparticles (NPs) of $\mathrm{CsPbI}_{3}$ are more stable. ${ }^{9,10}$ An interesting option is to use a perovskite composition having two cations at the A site, the so-called mixed cation perovskites. ${ }^{11}$ The mixed cation perovskites in their bulk form have achieved a high photovoltaic performance for several structures of solar cells. ${ }^{12,13,14}$ Another important feature of mixed cation perovskite is its ability to influence the band gap of the perovskite in a gentler way than the halides do, owing to modifying the angle at the $\mathrm{X}-\mathrm{Pb}-\mathrm{X}$ framework. ${ }^{15,16}$

Most attention has been directed to investigating the bulk properties of metal lead halide perovskites, whereas scant efforts have been devoted to elucidating the nano-scale properties of the perovskite material. In the last few years, however, the synthesis and characterization of nanoscale inorganic and hybrid perovskites have rapidly progressed. ${ }^{17}$ The first synthesis of Cs lead halide was reported by Protesescu et al. ${ }^{18} \mathrm{Cs}$ lead halide perovskite NPs have a direct band gap. The tunability of their band gap energies is feasible via altering the composition by changing the halides, $\mathrm{Cl}, \mathrm{Br}$, and $\mathrm{I}$. In addition, it was reported that the anion exchange could also be applied to control the absorption wavelength. ${ }^{19,20}$ The diameter of Cs lead halide perovskite NPs can be up to about 12-15nm; Cs lead halide perovskite NPs therefore exhibit an energy gap tunability in the visible spectrum $\left(400 \mathrm{~nm}-700 \mathrm{~nm}\right.$ wavelength). $\mathrm{CsPbX}_{3} \mathrm{NPs}$ have narrow emissions and high quantum yields up to $90 \% .^{21,22}$ To date, there have been reports on several $\mathrm{CsPbX}_{3} \mathrm{NP}$ synthesis and surface chemistry methods, including studying their optoelectronic properties. ${ }^{23,24,25}$

However, there have been only a few reports on mixed cation perovskite NPs in the nanoscale regime. A mixing of organic and inorganic cations (FA and $\mathrm{Cs}$ ) in perovskite NPs leads to FAdoped $\mathrm{CsPbI}_{3}$ with a cubic shape and uniform size of $10-15 \mathrm{~nm}$. These NPs exhibit better 
robustness than do the pure MA and pure Cs-based NPs. Their optical properties were tuned from the red to the NIR region. ${ }^{26}$ In addition, mixing two inorganic cations in the same NPs was recently reported by $\mathrm{us}^{27}$; Rb was incorporated into Cs-based NPs, forming all inorganic mixed cation perovskite NPs. The addition of Rb atoms slightly increases the NP band gap, shifting the NP absorbance and PL to shorter wavelengths. Moreover, NP's size decreases when $\mathrm{Rb}$ atoms are introduced into them.

However, obtaining a more detailed understanding of the Rb distribution inside the NPs is impossible using indirect characterization techniques such as optical absorption and PL. In this work we used high angle annular dark field (HAADF) imaging, combined with energy dispersive spectrometry (EDS) - spectrum imaging (SI) to study in detail the atomic distributions of the $\mathrm{Rb}$ and $\mathrm{Cs}$ atoms inside single perovskite NPs. It is a challenging task to analyze the perovskite materials with this high-resolution tool, since these materials are very sensitive to electron beams. We vary the $\mathrm{Rb}$ concentration within the $\mathrm{Rb}_{\mathrm{x}} \mathrm{Cs}_{1-\mathrm{x}} \mathrm{PbBr}_{3} \mathrm{NPs}$, when $\mathrm{X}=0,0.2,0.4,0.6$, and 0.8 including the case of pure Rb lead chloride NPs. Apparently the Rb atoms prefer to be located in the core of the nanoparticle; the Cs atoms remain on the nanoparticle's shell at medium Rb concentrations, whereas at higher Rb concentrations, a phase separation occurs.

\section{Results and discussion}

Here we present a detailed structural study of mixed inorganic cation lead halide perovskite NPs at the atomic level. We focused our investigations on mixed cation perovskite NPs having an $\mathrm{Rb}_{\mathrm{x}} \mathrm{Cs}_{1-\mathrm{x}} \mathrm{PbBr}_{3}$ composition (when $\mathrm{X}=0,0.2,0.4,0.6$, and 0.8 ). The HAADF detector was used to observe the atomic resolution images; EDS SI allows one to obtain detailed elemental maps of the NPs.

In our previous report we synthesized mixed cation perovskite NPs, along with the ability to tune their optical properties. When $\mathrm{Rb}$ atoms are introduced into the $\mathrm{CsPbr}_{3} \mathrm{NPs}$, a blue shift of the absorbance and photoluminescence (PL) is observed. For example, the PL wavelength of pure $\mathrm{Cs}(\mathrm{x}=0)$ is equal to $516 \mathrm{~nm}$, whereas when $\mathrm{X}=0.2$ (some $\mathrm{Rb}$ is introduced into the NP) the PL wavelength is shifted to $508 \mathrm{~nm}$ (table 1). Since the ionic radius of $\mathrm{Rb}^{+}$is smaller than that of $\mathrm{Cs}^{+}$, the perovskite's framework becomes distorted. In this work we went down to the atomic level, in order to analyze the atomic distribution and chemical composition of single particles. In particular, we detected the $\mathrm{Rb}$ to $\mathrm{Cs}$ ratios and their distribution over the NPs.

Using the STEM elemental line scan, it is possible to identify the elements as a function of their position inside the particle. The EDS elemental analysis is based on measuring the 
characteristic X-ray fluorescence emitted due to the interactions of high velocity electrons with sample atoms; each atom has a different signal due to the different energies of the atoms' shells. Notably the EDS analysis of $\mathrm{Rb}$ appears to be very challenging in the presence of $\mathrm{Cs}$ and $\mathrm{Pb}$ atoms. The main $\mathrm{Rb}$ signal derives from two shells: $\mathrm{L}(\sim 1.69 \mathrm{KeV})$ and $\mathrm{K}(\sim 13.37 \mathrm{KeV})$. The $\mathrm{Rb} \mathrm{L}$ shell overlaps with the $\mathrm{K}$ shell signal of $\mathrm{Si} \mathrm{K}(\sim 1.73 \mathrm{KeV}), \mathrm{Br} \mathrm{L}_{\gamma 2}(\sim 1.77 \mathrm{KeV})$, and the $\mathrm{L}_{\beta 3}(\sim 1.60 \mathrm{KeV})$ shell, whereas the $\mathrm{K}$ shell overlaps with both $\mathrm{Br} \mathrm{K}_{\beta}(\sim 13.29 \mathrm{KeV})$ and $\mathrm{Pb} \mathrm{L} \gamma$ $(\sim 14.75 \mathrm{KeV})$. Detection of small Rb traces by EDS is therefore almost impossible in such systems.

Figure 1 shows the case of pure $\mathrm{CsPbBr}_{3}$ NPs (i.e. $\mathrm{x}=0$ ). Figure 1a presents an atomic resolution HAADF STEM image of single NPs; the fast Fourier transform (FFT) of the image can be seen in the inset of Figure 1a. An analysis of the crystallographic structure of the NPs, using the FFT, is presented in Table 1, which shows the d-spacing values and the corresponding Miller indices $\left(\begin{array}{lll}5 & 0 & 0\end{array}\right)$ and ( $\left.\begin{array}{ll}4 & 4\end{array}\right)$. The arrow in Figure 1c indicates the integrated line scan direction; Figure $1 \mathrm{~d}$ shows the detected elements as a function of the distance.

It can be seen that there is no clear difference exists between the intensities of the elements. In this NP orientation (zone axis [100]) the Cs atoms are ordered as a monoatomic column; $\mathrm{Pb}$ and $\mathrm{Br}$ lead to mixed columns and finally, $\mathrm{Br}$ monoatomic columns are also present. However, $\mathrm{Pb}$ and $\mathrm{Br}$ can hardly be recognized because $\mathrm{Br}$ is the lighter element in the structure.
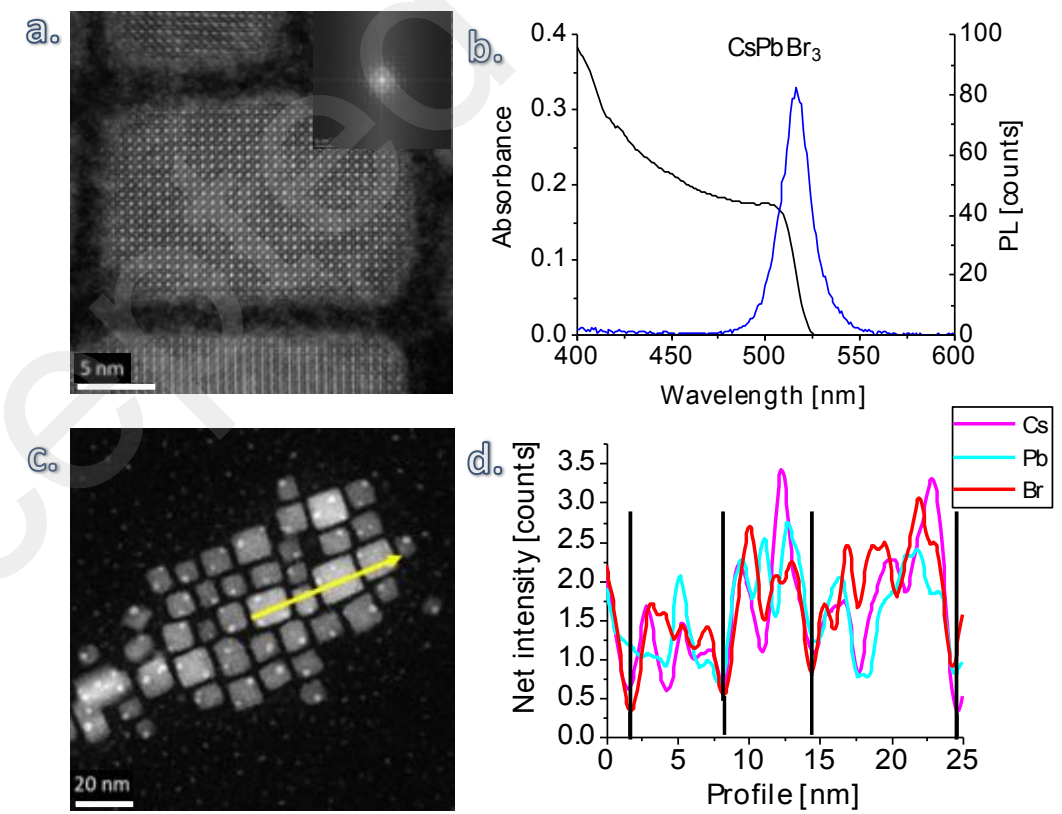

Figure 1: (a) STEM image of $\mathrm{CsPbBr}_{3}$ NPs with atomic resolution. Inset: FFT of a single particle. (b) Absorbance and photoluminescence spectra of the $\mathrm{CsPbBr}_{3}$ NPs. (c) HAADF STEM image; the arrow denotes the scanned area. (d) The arrow in Figure 1c denotes the chemical elemental analysis as a function of the distance. The black lines denote where each NP starts and ends. 
Increasing the amount of $\mathrm{Rb}$ in the NPs results in mixed inorganic cation perovskite NPs, as shown in Figure 2, when $\mathrm{X}=0.2$ where $\mathrm{Rb}_{0.2} \mathrm{Cs}_{0.8} \mathrm{PbBr}_{3}$. Here the PL shifts slightly to shorter wavelengths compared with pure Cs NPs (508 nm compared to $516 \mathrm{~nm}$ ), as presented in Table 1 and Figure $2 b$.

With low $\mathrm{Rb}$ concentrations, it is very difficult to detect the $\mathrm{Rb}$ signal. We performed $\mathrm{a}$ reference experiment in which we tracked the $\mathrm{Rb}$ signal from pure Cs-based NPs (see Figure $1 \mathrm{~S}$ in the supporting information). The $\mathrm{Rb}$ signal is within the error limit of the measurements for both $X=0$ and $X=0.2$; Figure $2 d$ shows that the $R b$ signal is five times smaller than the $C$ s signal.

Despite the low $\mathrm{Rb}$ signals, the presence of the $\mathrm{Rb}$ atoms is indicated, as can be seen in the elemental map in Figure 2c, which shows green dots located mainly inside the NP.

Figure 2a shows HAADF STEM images and a FFT pattern of a single NP. The FFT provides the Miller indices (4 22 ) and (4 4 4) corresponding to $\mathrm{Rb}_{0.2} \mathrm{Cs}_{0.8} \mathrm{PbBr}_{3}$. Importantly, the HAADF STEM image shows some white spots within the NPs. T. Udayabhaskararao et al. ${ }^{28}$ associated these spots with $\mathrm{Pb}^{0} \mathrm{NPs}$. In this work, using HADDF the white spots could also be associated with $\mathrm{Pb}^{0}$ particles (Figure S2).
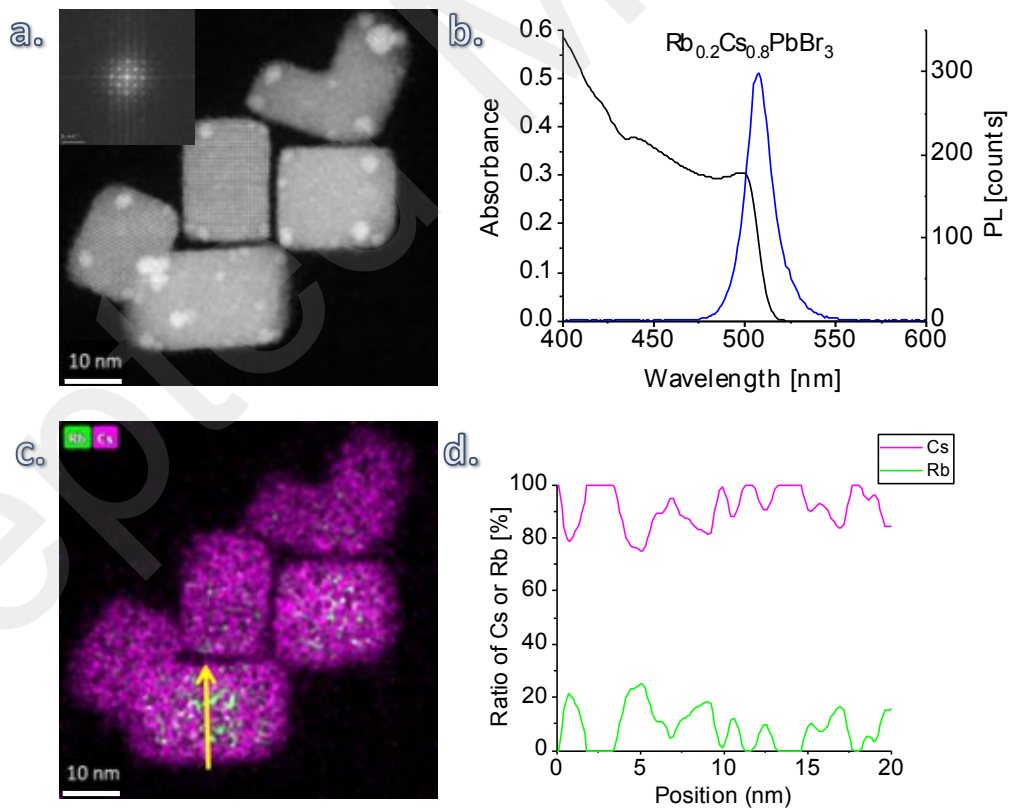

Figure 2: (a) HAADF STEM of $\mathrm{Rb}_{0.2} \mathrm{Cs}_{0.8} \mathrm{PbBr}_{3}$ NPs. Inset: FFT of a single particle. (b) Absorbance and photoluminescence spectra of $\mathrm{Rb}_{0.2} \mathrm{Cs}_{0.8} \mathrm{PbBr}_{3}$ NPs. (c) EDS maps of $\mathrm{Rb}$ and $\mathrm{Cs} ; \mathrm{Rb}$ is denoted in green and $\mathrm{Cs}$ in magenta. The arrow shows the integrated profile as presented in Figure d. (d) $\mathrm{Rb} /(\mathrm{Cs}+\mathrm{Rb})$ and $\mathrm{Cs} /(\mathrm{Cs}+\mathrm{Rb})$ ratios as a function of the distance. 
Figure 3 shows the case of a higher $\mathrm{Rb}$ concentration, $\mathrm{Rb}_{0.4} \mathrm{Cs}_{0.6} \mathrm{PbBr}_{3}$, when $\mathrm{X}=0.4$. Here, owing to the larger amount of $\mathrm{Rb}$, it is possible to observe by elemental analysis the two cations (i.e., $\mathrm{Cs}^{+}$and $\mathrm{Rb}^{+}$) in the same NP. The $\mathrm{Rb}$ signal can be easily recognized and it is equal in intensity to that of Cs.

Figure $3 \mathrm{a}$ shows the HAADF image of the NPs when $\mathrm{X}=0.4 \mathrm{Rb}$ and with 0.6 of $\mathrm{Cs}$; the inset presents the FFT of a single NP; this provides us with the d-spacing values and the corresponding Miller indices ( $\begin{aligned} & 3 \\ & 3\end{aligned} 2$ ) and (4 5 2) (see Table 1). Importantly, when $X=0.4$ and $\mathrm{X}=0.6$, the samples show a high stability under the HR-TEM electron beam.

The PL and absorbance when $X=0.4$ (Figure $3 b$ ) indicate that $\lambda_{\max }=496 \mathrm{~nm}$, which is further blue-shifted in relation to pure $\mathrm{CsPbBr}_{3}$. The elemental composition of a single NP is observed in Figures $3 \mathrm{c}$ and $3 \mathrm{~d}$. The arrow denotes the scanned area, which is shown in the graph in Figure $3 \mathrm{~d}$. The ratios of $\mathrm{Cs} /(\mathrm{Cs}+\mathrm{Rb})$ and $\mathrm{Rb} /(\mathrm{Cs}+\mathrm{Rb})$ are presented in Figure $3 \mathrm{~d}$, which clearly shows a mirror image of $\mathrm{Rb}$ and Cs. As shown in Figure 3d, a Cs atom is replaced by a Rb atom and vice versa. Interestingly, based on the elemental map in Figure 3c, the Rb atoms are located in the center of the NP, whereas the Cs atoms are located on the surface of the NP, forming a "core-shell"-like structure. With larger particles (i.e., 10-12nm), it is easier to recognize the "core-shell" structure, whereas with small NPs the signal is weaker and the particles are thinner, which makes it harder to recognize this structure, even though $\mathrm{Rb}$ appears to be at the inner region of NP and Cs on the NP shell .

The pure Rb-based lead bromide perovskite is not stable as bulk materials as well as NPs are, as reported previously ${ }^{3}$. On the other hand, $\mathrm{CsPbrr}_{3} \mathrm{NPs}$ have a stable perovskite phase and can be easily synthesized. Moreover, the high surface energy of NP forces its shell to be more energetically stable than its core. Therefore, in order to obtain mixed cation NPs that are stable, Cs will preferably be located on the shell of the NP; Rb will be located in the core of the NP in order to stabilize this composite structure.

In order to support this observation, density functional theory (DFT) modeling ${ }^{29,30}$ was performed (see the computational details in the SI). The modeling starts from the bulk structures of $\mathrm{CsPCl}_{3}$ and $\mathrm{CsPbBr}_{3}$ by optimizing them until the residual forces are below $0.005 \mathrm{eV} / \AA$. The models were constructed to illustrate the substitution effect of $\mathrm{Cs}$ by $\mathrm{Rb}$ cations in $\mathrm{CsPCl}_{3}$. A supercell of 98 atoms with a vacuum of $200 \AA$ was considered by using the SIESTA package. ${ }^{31,32}$ Three types of substitution in the initial supercell have been simulated, mimicking $\mathrm{X}=0.125,0.25$, and 0.375 concentrations; they are hereafter referred to as $7 \mathrm{Cs}: 1 \mathrm{Rb}, 6 \mathrm{Cs}: 2 \mathrm{Rb}$, and $5 \mathrm{Cs}: 3 \mathrm{Rb}$, respectively. As discussed in the SI, each case leads to a 
stable structure. Figure $6 \mathrm{~b}$ shows side views of the three most stable structures having three different compositions. When $\mathrm{X}=0.125$ (7Cs:1Rb) the simulation shows that a single $\mathrm{Rb}$ cation prefers to be located far from the surface $(E=-263.5 \mathrm{meV})$. For $\mathrm{X}=0.25(6 \mathrm{Cs}: 2 \mathrm{Rb})$ a pair of $\mathrm{Rb}$ cations again prefers to be far from the surface but in a position somewhat closer to the surface than a single $\mathrm{Rb}$ cation $(\mathrm{E}=-227.5 \mathrm{meV})$ is. Lastly, when $\mathrm{X}=0.375$ (5Cs:3Rb), three $\mathrm{Rb}$ cations were introduced in the supercell and pos5 was found to be the most stable configuration $(\mathrm{E}=$ $176.1 \mathrm{meV})$. In fact, it confirms the tendency shown by 1 and then 2 substitutions. The case of $5 \mathrm{Cs}: 3 \mathrm{Rb}$ indicates that a stable structure can be achieved by combining a single $\mathrm{Rb}$ cation in the core of the particle and a pair of $\mathrm{Rb}$ cations on the subsurface. This result again supports the experimental observations of cases when $\mathrm{X}=0.4$ and $\mathrm{X}=0.6$, where the $\mathrm{Rb}$ atoms prefer to be far from the surface of the nanoparticle.
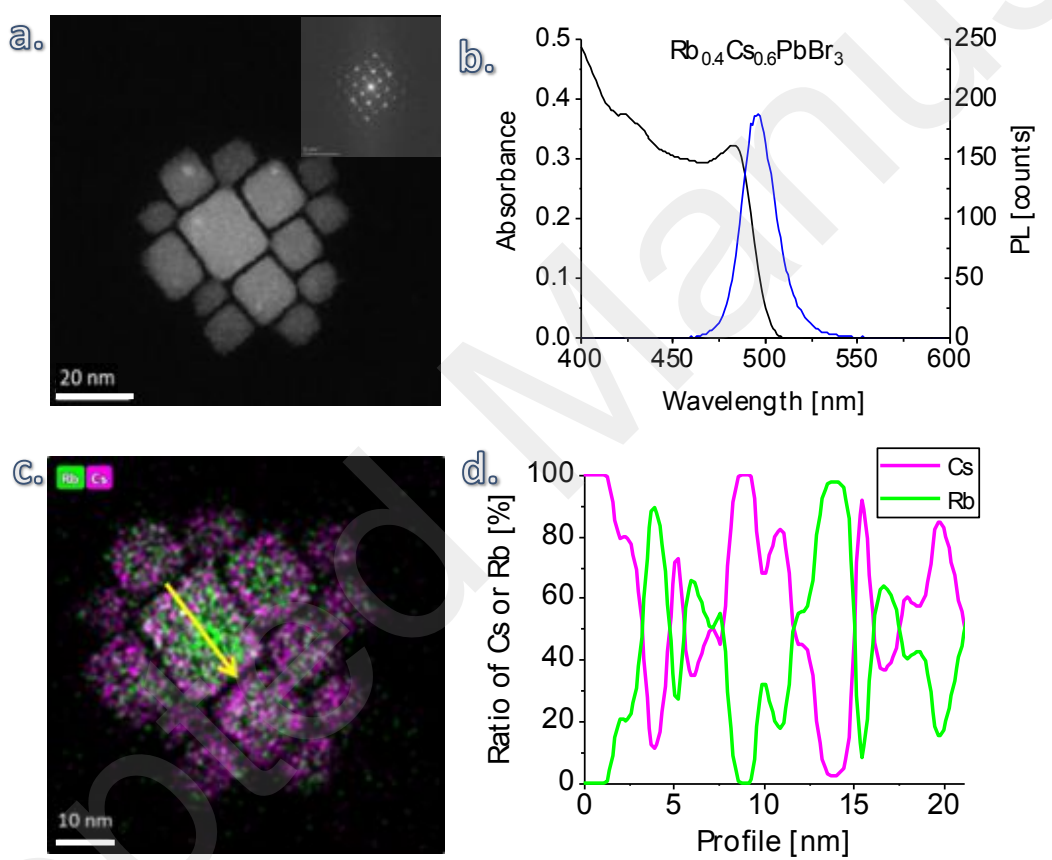

Figure 3: (a) HAADF STEM of $\mathrm{Rb}_{0.4} \mathrm{Cs}_{0.6} \mathrm{PbBr}_{3}$ NPs. Inset: FFT of a single particle. (b) Absorbance and photoluminescence spectra of the $\mathrm{Rb}_{0.4} \mathrm{Cs}_{0.6} \mathrm{PbBr}_{3}$ NPs. (c) EDS maps indicating $\mathrm{Rb}$ in green and $\mathrm{Cs}$ in magenta. The arrow shows the profile position as presented in Figure d. (d) $\mathrm{Rb} /(\mathrm{Cs}+\mathrm{Rb})$ and $\mathrm{Cs} /(\mathrm{Cs}+\mathrm{Rb})$ ratios as a function of the distance.

Regarding $\mathrm{Rb}$, when $\mathrm{X}=0.6$ with absorbance $\left(\lambda_{\max }=491 \mathrm{~nm}\right)$, a slightly blue-shifted PL appears, as can be seen in Figure $4 \mathrm{~b}$. The atomic resolution HAADF image and the corresponding FFT are observed in Figure 4a. The Miller indices for this composition are indicated in Table 1. The same occurs when $\mathrm{X}=0.4$; also here the $\mathrm{Rb}$ phase is located at the core of the NP, whereas the Cs phase is located in the shell, as can be clearly seen in Figures $4 \mathrm{c}$ and $4 \mathrm{~d}$. The arrow in Figure $4 \mathrm{c}$ corresponds to the line scan in Figure 4d, which indicates the higher concentration of Cs in 
the NP shell, compared with Rb in the NP core. In addition, here Cs energetically prefers to be located in the NP shell and Rb in the NP core, as supported by the DFT calculations.
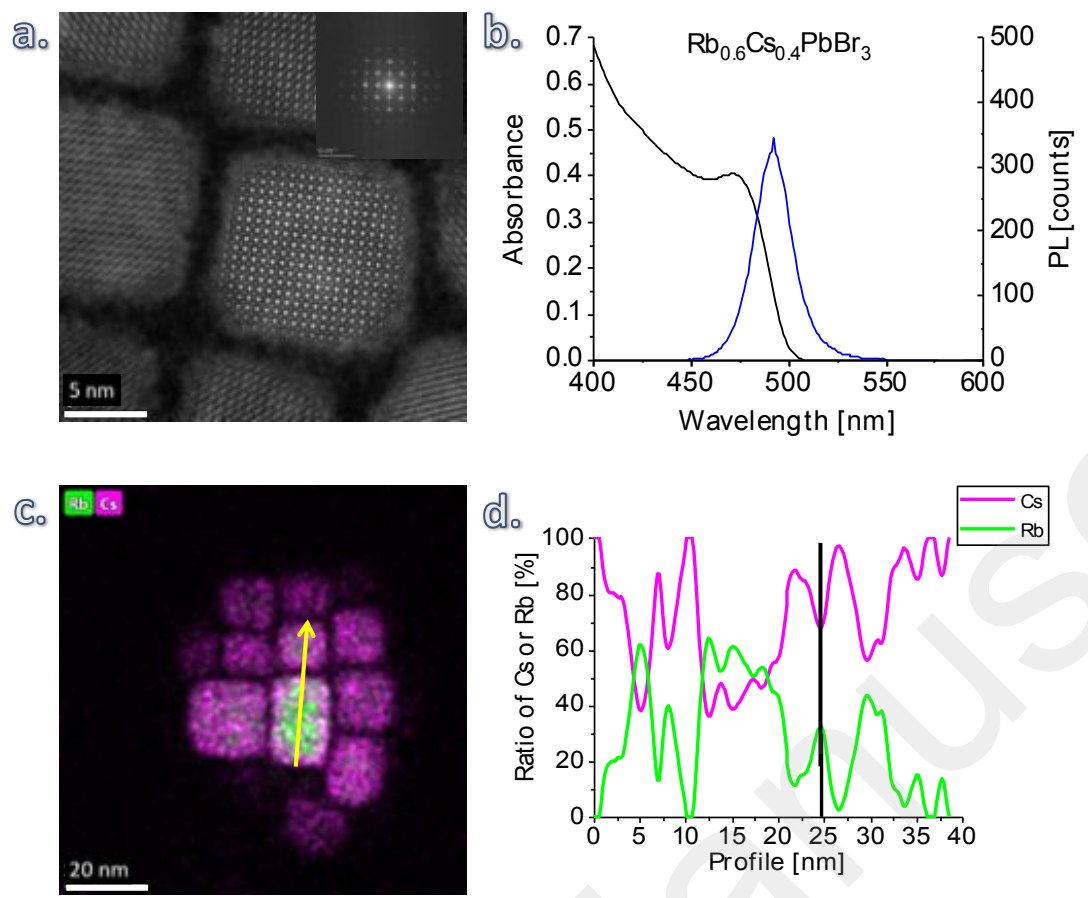

Figure 4: (a) $\mathrm{Rb}_{0.6} \mathrm{Cs}_{0.4} \mathrm{PbBr}_{3} \mathrm{NPs}$ with atomic resolution (by a HAADF detector). Inset: FFT of a single particle. (b) Absorbance and photoluminescence spectra of the $\mathrm{Rb}_{0.6} \mathrm{Cs}_{0.4} \mathrm{PbBr}_{3} \mathrm{NPs}$. (c) EDS in the TEM denoting $\mathrm{Rb}$ in green and $\mathrm{Cs}$ in magenta. The arrow shows the EDS profile as presented in Figure d. (d) The $\mathrm{Rb} /(\mathrm{Cs}+\mathrm{Rb})$ and $\mathrm{Cs} /(\mathrm{Cs}+\mathrm{Rb})$ ratios as a function of the distance. The black line denotes where one NP is and the other starts.

Increasing the $\mathrm{Rb}$ concentration to $\mathrm{X}=0.8$ results in additional PL blue-shifts and absorbance $\left(\lambda_{\max }=468 \mathrm{~nm}\right.$, Figure 5b). The STEM image in Figure 5a shows white spots located within a single NP; this indicates phase separation, which may appear due to the large amount of Rb compared to Cs. This is similar to when $\mathrm{X}=0.2$, where white spots were also be observed. However, here, since $\mathrm{Rb}$ alone cannot form a stable perovskite phase, it begins to separate from NP. It is unlikely that both $\mathrm{Cs}$ and $\mathrm{Rb}$ will be present in the same NP. In the case of $\mathrm{X}=0.2$ due to the small amount of $\mathrm{Rb}$, some of it can remain inside the NP. Figure $5 \mathrm{c}$ shows the existence of phase separation, where green (which denotes $\mathrm{Rb}$ ) particles and magenta (which denotes $\mathrm{Cs}$ ) particles, are separated from each other.

In large concentrations of $\mathrm{Rb}$, we expect to see $\mathrm{Rb}$ in the NP shell and $\mathrm{Cs}$ in the NP core, since the nucleation begins with $\mathrm{Rb}$, which is in excess. However, the amount of $\mathrm{Cs}$ is too small in a single NP; therefore, it cannot stabilize the structure that supports the observed phase separation. Moreover, a line scan on a single NP (Figures $5 \mathrm{c}$ and $5 \mathrm{~d}$ ) indicates that most of the 
Cs is located in the middle of the NP, whereas Rb is more on the NP's surface. This is also nicely illustrated by the DFT modeling; Pos1 in Figure S8 shows a case where three Rb cations are located on the NP surface; this results in the most unstable structure compared with the other cases.
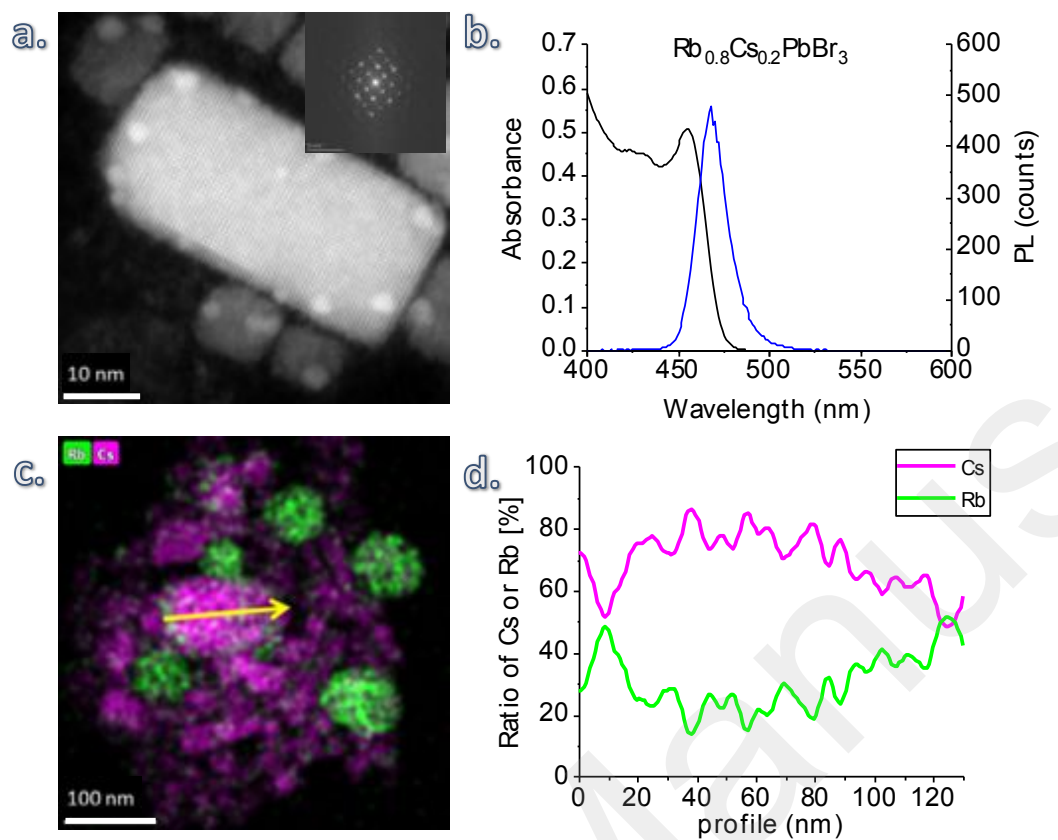

Figure 5: (a) $\mathrm{Rb}_{0.8} \mathrm{Cs}_{0.2} \mathrm{PbBr}_{3} \mathrm{NPs}$ (HAADF). Inset: FFT of a single particle. (b) Absorbance and photoluminescence spectra of $\mathrm{Rb}_{0.8} \mathrm{Cs}_{0.2} \mathrm{PbBr}_{3} \mathrm{NPs}$. (c) EDS in the TEM; $\mathrm{Rb}$ is denoted in green and $\mathrm{Cs}$ in magenta. The arrow shows the scanned area, as presented in Figure d. (d) The $\mathrm{Rb} /(\mathrm{Cs}+\mathrm{Rb})$ and $\mathrm{Cs} /(\mathrm{Cs}+\mathrm{Rb})$ ratios as a function of the distance.

Lastly, pure Rb-based NPs were synthesized and studied. The synthesis was based on our previous report ${ }^{33}$ on $\mathrm{Rb}_{6} \mathrm{~Pb}_{5} \mathrm{Cl}_{16}$. The $\mathrm{Rb}$ lead halide NPs cannot be synthesized with bromide (at the halide position) due to the larger ionic radius of bromide, compared with the chloride ion. Figure 6a shows a single $\mathrm{Rb}_{6} \mathrm{~Pb}_{5} \mathrm{Cl}_{16} \mathrm{NP}$ and its corresponding FFT. The white spots can be observed already in a single NP; the STEM image in Figure 6c shows several white spots for each NP, as was reported by us previously ${ }^{29}$. The $\mathrm{Rb}_{6} \mathrm{~Pb}_{5} \mathrm{Cl}_{16} \mathrm{NPs}$ form a tetragonal phase, which is a non-perovskite phase based on our X-ray diffraction analysis ${ }^{33}$. Monzel et al. ${ }^{34}$ reported on this phase; it was shown that the perovskite phase $\mathrm{RbPbCl}_{3}$ cannot exist at room temperature, whereas the $\mathrm{Rb}_{6} \mathrm{~Pb}_{5} \mathrm{Cl}_{16}$ phase can. The only option to observe a tetragonal perovskite phase based on $\mathrm{RbPbCl}_{3}$ is heating above $320^{\circ} \mathrm{C}$; however, below that temperature this phase does not exist. At higher temperatures, a cubic perovskite phase can also be observed. Based on our previous report, ${ }^{33}$ the white spots located on each NP are associated with the $\mathrm{Pb}^{0}$ or $\mathrm{Pb}_{2} \mathrm{O}_{3}$ phases. These white spots already appear in fresh samples of $\mathrm{Rb}_{6} \mathrm{~Pb}_{5} \mathrm{Cl}_{16}$ 
NPs, as opposed to when $\mathrm{X}=0,0.2$, and 0.8 ; these white spots usually appear because of the electron beam radiation.

Interestingly, it is worth mentioning that this $\mathrm{Rb}_{6} \mathrm{~Pb}_{5} \mathrm{Cl}_{16}$ structure was also observed with pure $\mathrm{CsPbBr}_{3} \mathrm{NPs}$; here Cs was used instead of the $\mathrm{Rb}$ cation, i.e., $\mathrm{Cs}_{6} \mathrm{~Pb}_{5} \mathrm{Br}_{16}$. This is the first time that this phase was observed in the synthesis of $\mathrm{CsPbBr}_{3}$ NPs (see Figure S4). Clearly, owing to the bigger size of Cs compared with $\mathrm{Rb}$, this structure can be formed with $\mathrm{Br}$ instead of $\mathrm{Cl}$, as in the case of $\mathrm{Rb}$. This interesting structure opens the way to investigate new phases of cesium lead bromide.

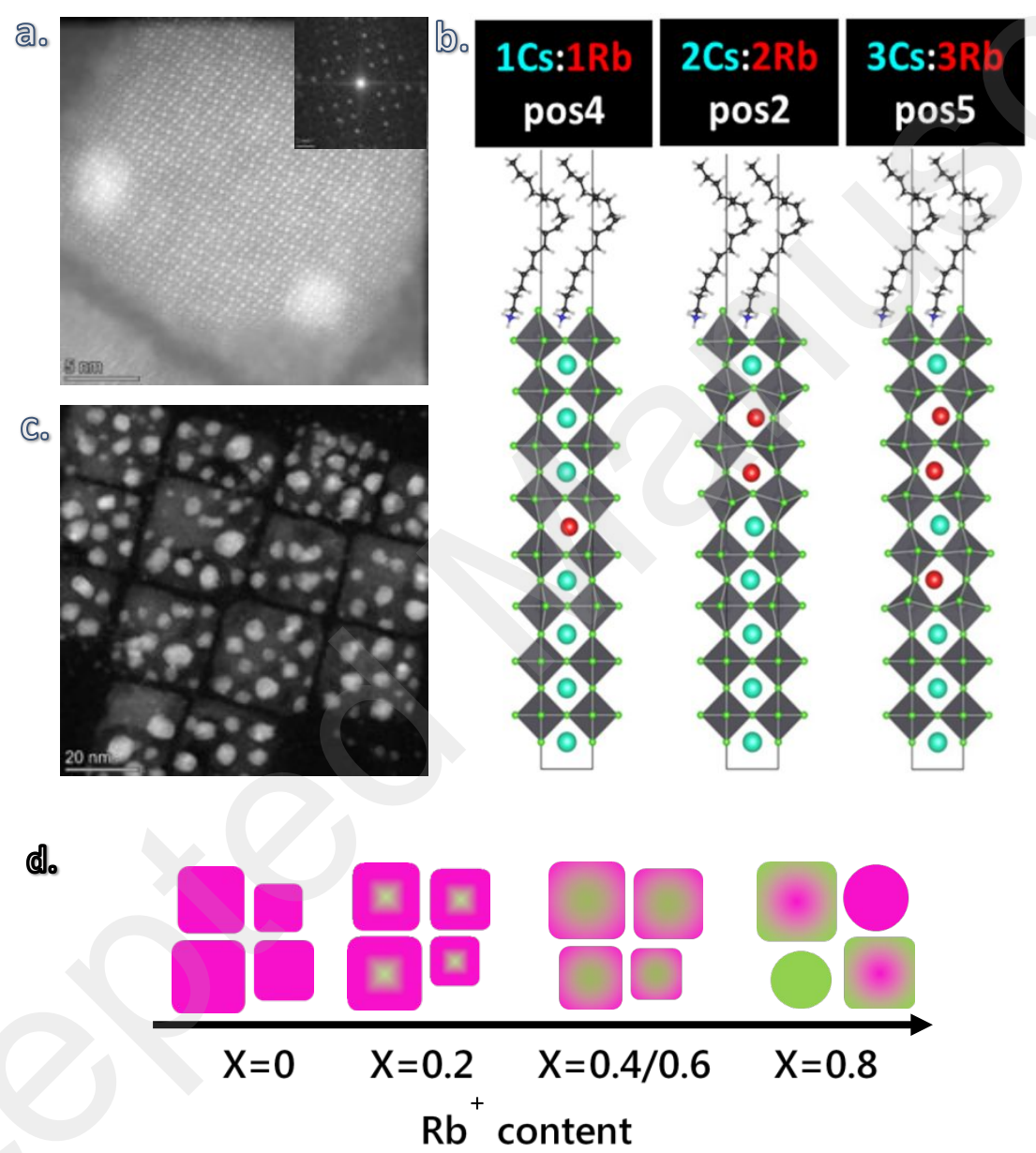

Figure 6: (a) HR-TEM image of single $\mathrm{Rb}_{6} \mathrm{~Pb}_{5} \mathrm{Cl}_{16} \mathrm{NP}$ and the corresponding electron diffraction (inset). (b) Side profiles for the three most stable structures of three different compositions. The cases $1 \mathrm{Cs}: 1 \mathrm{Rb}, 2 \mathrm{Cs}: 2 \mathrm{Rb}$, and $3 \mathrm{Cs}: 3 \mathrm{Rb}$ correspond to when $\mathrm{X}=0.125$, $\mathrm{X}=0.25$, and $\mathrm{X}=0.375$, respectively and with $\mathrm{Rb}_{\mathrm{x}} \mathrm{Cs}_{(1-\mathrm{x})} \mathrm{PbY}_{3}(\mathrm{Y}=\mathrm{Cl}, \mathrm{Br}$ ). (c) HR-TEM of $\mathrm{Rb}_{6} \mathrm{~Pb}_{5} \mathrm{Cl}_{16} \mathrm{NPs}$; the white spots indicate other phases present in these NPs. (d) Schematic illustration of increasing Rb concentration in the NPs. Magenda represent the $\mathrm{Cs}^{+}$and green the $\mathrm{Rb}^{+}$.

Figure $6 \mathrm{~d}$ summarizes the observation from the different $\mathrm{Rb}^{+}$concentrations in the NPs. Starting, from $\mathrm{X}=0$ which refers to the pure $\mathrm{CsPbBr}_{3}$, until the $\mathrm{X}=0.8$ where the $\mathrm{Rb}^{+}$ concentration is much higher than the $\mathrm{Cs}^{+}$concertation which possess phase separation. 
Figure S3 shows the full width half maximum (FWHM) based on the PL spectra of the different $\mathrm{Rb}$ concentrations. The PL measurement detects a macro size area of the sample, which means that it takes into account the PL from many NPs. Keeping this in mind, Figure S3 shows that in both the cases when $\mathrm{X}=0.4$ and $\mathrm{X}=0.6$ the FWHM is larger than the other concentrations. Both concentrations show the "core-shell" structure, which clearly indicates that Rb is incorporated inside the NPs, whereas in the other concentrations Rb was hardly detected or was phase separated. Therefore, when $\mathrm{X}=0,0.2$, and 0.8 , the FWHM value is smaller, since it mainly detects the pure Cs lead bromide NPs, whereas when $\mathrm{X}=0.4$ and 0.6 , it detects the coreshell particles that contain $\mathrm{Rb}$ and $\mathrm{Cs}$ together in the same NPs; therefore, the FWHM increases. This observation indicates the importance of analyzing single NPs at their atomic level.

Table 1: Miller indices, the PL max and the full width half maximum (FWHM) for different additions of $\mathrm{Rb}$.

\begin{tabular}{|c|c|c|c|c|c|c|}
\hline Added Rb & $\begin{array}{c}\text { d-spacing } \\
(\AA)\end{array}$ & (h k l) & $\begin{array}{c}\text { d-spacing } \\
(\AA)\end{array}$ & (h k l) & $\lambda_{\max }(\mathbf{n m})$ & $\begin{array}{c}\text { FWHM } \\
(\mathrm{nm})\end{array}$ \\
\hline$X=0$ & $1.17 \pm 0.02$ & $\left(\begin{array}{lll}5 & 0 & 0\end{array}\right)$ & $0.847 \pm 0.01$ & $(444)$ & 516 & 17.7 \\
\hline$X=0.2$ & $1.19 \pm 0.02$ & $\left(\begin{array}{lll}4 & 2 & 2\end{array}\right)$ & $0.842 \pm 0.01$ & $(444)$ & 508 & 17.0 \\
\hline$X=0.4$ & $1.26 \pm 0.02$ & $\left(\begin{array}{lll}3 & 3 & 2\end{array}\right)$ & $0.874 \pm 0.01$ & $\left(\begin{array}{lll}4 & 5 & 2\end{array}\right)$ & 496 & 21.0 \\
\hline $\mathrm{X}=0.6$ & $1.20 \pm 0.02$ & $\left(\begin{array}{lll}4 & 2 & 2\end{array}\right)$ & $0.854 \pm 0.01$ & $(444)$ & 491 & 21.4 \\
\hline $\mathrm{X}=0.8$ & $1.2 \pm 0.02$ & $\left(\begin{array}{lll}4 & 2 & 2\end{array}\right)$ & $0.866 \pm 0.01$ & $\left(\begin{array}{lll}6 & 3 & 1\end{array}\right)$ & 468 & 18.0 \\
\hline
\end{tabular}

\section{Experimental \\ Chemicals}

Cesium carbonate $\left(\mathrm{Cs}_{2} \mathrm{CO}_{3}, 99.9 \%\right.$, Sigma-Aldrich), rubidium carbonate $\left(\mathrm{Rb}_{2} \mathrm{CO}_{3}, 99 \%\right.$, Sigma-Aldrich), lead (II) chloride ( $\mathrm{PbCl}_{2}, 98 \%$, Sigma-Aldrich), lead(II) bromide ( $\mathrm{PbBr}_{2}$, $\geq 98 \%$, Sigma-Aldrich), oleic acid (OA, 90\%, Sigma-Aldrich), oleylamine (OLAM, 70\%, Sigma-Aldrich), 1-octadecene (ODE, 90\%, Sigma-Aldrich), 2-propanol ( $\geq 99.8 \%$, SigmaAldrich), trioctylphosphine (TOP, 97\%, Strem), and hexane (not pure, Gadot) were purchased and used as received, without any further purification.

\section{Preparation of $\mathbf{R b} / \mathbf{C s}-$ oleate}

$\mathrm{The} \mathrm{Rb} / \mathrm{Cs}$-oleate precursor was prepared according to a previously published procedure by Protesescu et al. and by us ${ }^{18,27} \mathrm{In}$ a $100 \mathrm{~mL}$ 3-neck flask, different molar ratios of $\mathrm{Cs}_{2} \mathrm{CO}_{3} / \mathrm{Rb}_{2} \mathrm{CO}_{3}$ (total of $1.228 \mathrm{mmol}$ ) were mixed with $625 \mu \mathrm{L}$ of oleic acid $(\mathrm{OA})$ and $7.5 \mathrm{~mL}$ 
of 1-octadecene (ODE). The solution was degassed for $1 \mathrm{~h}$ under vacuum conditions at $120{ }^{\circ} \mathrm{C}$ and then heated to $150{ }^{\circ} \mathrm{C}$ under Argon flow.

\section{Synthesis of $\mathrm{Rb}_{x} \mathrm{Cs}_{1-x} \mathrm{PbX}_{3}(\mathrm{X}=\mathrm{Cl}, \mathrm{Br}) \mathrm{NPs}$}

The NPs were synthesized according to our previous report. ${ }^{27}$ First, $0.188 \mathrm{mmol}^{\circ} \mathrm{PbX}_{2}$ was mixed with $0.5 \mathrm{~mL}$ of OA, $0.5 \mathrm{~mL}$ of OLA, and $5 \mathrm{~mL}$ of ODE in an additional $100 \mathrm{~mL}$ 3-neck flask (for $\mathrm{X}=\mathrm{Cl}, 1 \mathrm{ml}$ of TOP was added to the flask). The solution was degassed for $1 \mathrm{~h}$ under vacuum at $120^{\circ} \mathrm{C}$ and then heated to $150{ }^{\circ} \mathrm{C}$ under an Argon flow. The reaction was carried out by injecting $0.4 \mathrm{~mL}$ of the $\mathrm{Rb} / \mathrm{Cs}$-oleate precursor solution into the $\mathrm{PbX}_{2}$ precursor solution using a preheated syringe. The reaction was quenched using an ice bath after a few seconds. Isopropanol was added to the crude solution in a volume ratio of $2: 1$ and the NCs were centrifuged at $6000 \mathrm{rpm}$ for $10 \mathrm{~min}$, the participate was dispersed in hexane, isopropanol was added again in a volume ratio of $1: 1$, and the NCs were centrifuged again at $6000 \mathrm{rpm}$ for 10 min. The purified NCs were dispersed in hexane for further characterization.

\section{Synthesis of $\mathrm{Rb}_{6} \mathrm{~Pb}_{5} \mathrm{Cl}_{16} \mathrm{NPs}$}

$\mathrm{Rb}_{6} \mathrm{~Pb}_{5} \mathrm{Cl}_{16}$ NPs were synthesized according to our previously reported procedure. ${ }^{33}$ Briefly, $0.138 \mathrm{~g}$ of $\mathrm{Rb}_{2} \mathrm{CO}_{3}$ was mixed with $625 \mu \mathrm{l}$ of OA and $7.5 \mathrm{ml}$ of ODE in a $100 \mathrm{ml}$ three-neck flask. The solution was degassed for $1 \mathrm{~h}$ under vacuum at $120^{\circ} \mathrm{C}$ and then heated to $150{ }^{\circ} \mathrm{C}$ under argon flow.

Next, $0.052 \mathrm{~g}$ of $\mathrm{PbCl}_{2}$ was mixed with $0.5 \mathrm{ml}$ of OA, $0.5 \mathrm{ml}$ of OLA, $1 \mathrm{ml}$ of TOP, and $5 \mathrm{ml}$ of ODE in an additional $100 \mathrm{ml}$ three-neck flask. The mixture was degassed for $1 \mathrm{~h}$ under vacuum at $120^{\circ} \mathrm{C}$ and then heated to $150{ }^{\circ} \mathrm{C}$ under argon flow. Then $0.4 \mathrm{ml}$ of the $\mathrm{Rb}$-oleate precursor solution was injected into the $\mathrm{PbCl}_{2}$ precursor solution. The reaction was quenched after a few seconds using an ice bath. Isopropanol was added to the crude solution in a volume ratio of $1: 1$ and the NCs were centrifuged at $6000 \mathrm{rpm}$ for $10 \mathrm{~min}$. The last stage was repeated once and then the purified NCs were dispersed in hexane for further characterization.

\section{High-resolution transmission electron microscopy (HRTEM)}

Scanning TEM high angle annular dark field (STEM-HAADF) images and the FFT of atomic resolution images were captured with a probe-corrected high-resolution scanning transmission electron microscope, Themis Z, produced by Thermo Fisher Scientific, and operated at an accelerating voltage of $300 \mathrm{Kv}$ (it can also operate at $80 \mathrm{Kv}$ ). Elemental analysis of NPs was performed with a Super-X EDS (Energy Dispersive X-Ray Spectroscopy) detector.

Samples were prepared as follows: $2 \mu \mathrm{L}$ of the NC dispersion were dropped on a thin copper grid coated with an ultra-thin amorphous carbon film on holey carbon, and then the solvent was evaporated using a vacuum chamber. 


\section{Optical measurements}

Absorbance spectra were recorded using a Jasco V-670 spectrophotometer. Photoluminescence (PL) measurements were performed using an L-shaped spectrofluorometer (Edinburgh Instruments FL920). The Cl- and Br-based NPs were excited at 320 and $400 \mathrm{~nm}$, respectively. The emission was collected at $90^{\circ}$ in the range of 350-450 nm for $\mathrm{Cl}$ and $400-600 \mathrm{~nm}$ for $\mathrm{Br}$.

\section{Computational Details}

The computational details can be found in the supporting information.

\section{Acknowledgments}

This work was supported within the framework of the Israeli-French scientific cooperation (Joint Research Projects 2019-2021 entitled: ALLPOA (From Atomic Level to layered Perovskite for Optoelectronic Applications)) by the Ministry of Science \& Technology of the State of Israel (MOST) and France's Centre National de la Recherche Scientifique (CNRS).

The ab initio simulations were performed on HPC resources of TGCC and CINES under allocation 2019-A0060906724 sponsored by GENCI (Grand Equipement National de Calcul Intensif). L.E. would like to acknowledge the Israel Science Foundation grant No. 937/18. J. E. is a senior member of the Institut Universitaire de France.

\section{Conclusions}

In this work, we synthesized full inorganic mixed cation perovskite NPs. We introduced $\mathrm{Cs}^{+}$ and $\mathrm{Rb}^{+}$cations at different concentrations into these NPs. STEM-HAADF and FFT analysis were used to obtain an atomic level resolution of these NPs. It was found that in cases when $\mathrm{X}=0.4$ and $0.6, \mathrm{Rb}$ is located in the core of the nanoparticle, whereas Cs was observed in the shell. Increasing the concentration to $X=0.8$ revealed that phase separation exists between $\mathrm{Rb}$ and Cs. DFT calculations showed that a stable nanoparticle is observed when $\mathrm{Rb}$ is located far from the NP surface; however, when Rb atoms are located on the NP surface, a non-stable structure is observed, which leads to phase separation. Moreover, pure Rb NPs were synthesized, forming a non-perovskite structure with $\mathrm{Pb}^{0}$ as the nucleation sites. This work reveals the importance of atomic level investigations to better understand the properties and stability of these fundamentally interesting mixed cation perovskite NPs. The research here can generally advance our understanding of the bulk mixed cation perovskites. Moreover, it can 
open the way for new directions to improve the stability of halide perovskites and control their properties.

\section{Supporting information}

Supporting information is available free of charge via the internet at http://pubs.acs.org. The supporting information includes: Additional data extracted from the STEM photos and the EDS elemental maps. A Detailed explanation of the theoretical calculations and theory and the calculated theoretical energies of $1 \mathrm{Cs}: 1 \mathrm{Rb}, 2 \mathrm{Cs}: 2 \mathrm{Rb}$ and $3 \mathrm{Cs}: 3 \mathrm{Rb}$ at different positions.

\section{TOC}

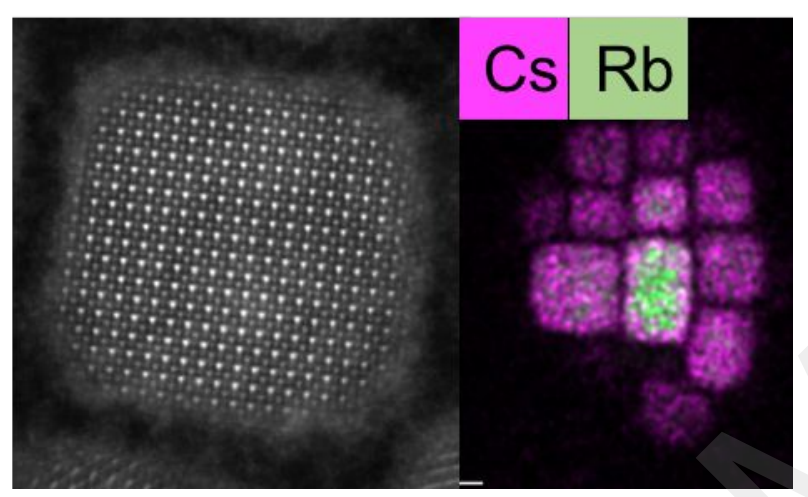

\section{References}

${ }^{1}$ NREL transforming energy. https://www.nrel.gov/pv/cell-efficiency.html (accessed Sep 26, 2019). Photovoltaic Research. Best Research-Cell Efficiency Chart

${ }^{2}$ Stranks, S. D.; Eperon, G. E.; Grancini, G.; Menelaou, C.; Alcocer, M. J. P.; Leijtens, T.; Herz, L. M.; Petrozza, A.; Snaith, H. J. Electron-Hole Diffusion Lengths Exceeding 1 Micrometer in an Organometal Trihalide Perovskite Absorber. Science 2013, 342 (6156), 341344. https://doi.org/10.1126/science.1243982

${ }^{3}$ Miyata, A.; Mitioglu, A.; Plochocka, P.; Portugall, O.; Wang, J. T.-W.; Stranks, S. D.; Snaith, H. J.; Nicholas, R. J. Direct Measurement of the Exciton Binding Energy and Effective Masses for Charge Carriers in Organic-inorganic Tri-Halide Perovskites. Nat. Phys. 2015, 11 (7), 582587. https://doi.org/10.1038/nphys3357.

${ }^{4}$ Filip, M. R.; Eperon, G. E.; Snaith, H. J.; Giustino, F. Steric Engineering of Metal-Halide Perovskites with Tunable Optical Band Gaps. Nat. Commun. 2014, 5 (1), 5757. https://doi.org/10.1038/ncomms6757.

${ }^{5}$ Eperon, G. E.; Stranks, S. D.; Menelaou, C.; Johnston, M. B.; Herz, L. M.; Snaith, H. J. Formamidinium Lead Trihalide: A Broadly Tunable Perovskite for Efficient Planar Heterojunction Solar Cells. Energy Environ. Sci. 2014, 7 (3), 982. https://doi.org/10.1039/c3ee43822h.

${ }^{6}$ Zhao, Y.; Nardes, A. M.; Zhu, K. Solid-State Mesostructured Perovskite $\mathrm{CH}_{3} \mathrm{NH}_{3} \mathrm{PbI}_{3}$ Solar Cells: Charge Transport, Recombination, and Diffusion Length. J. Phys. Chem. Lett. 2014, 5 (3), 490-494. https://doi.org/10.1021/jz500003v.

${ }^{7}$ Park, N.-G. Perovskite Solar Cells: An Emerging Photovoltaic Technology. Mater. Today 
2015, 18 (2), 65-72. https://doi.org/10.1016/J.MATTOD.2014.07.007.

${ }^{8}$ De Roo, J.; Ibáñez, M.; Geiregat, P.; Nedelcu, G.; Walravens, W.; Maes, J.; Martins, J. C.; Van Driessche, I.; Kovalenko, M. V.; Hens, Z. Highly Dynamic Ligand Binding and Light Absorption Coefficient of Cesium Lead Bromide Perovskite Nanocrystals. ACS Nano 2016, 10 (2), 2071-2081. https://doi.org/10.1021/acsnano.5b06295.

${ }^{9}$ Huang, H.; Bodnarchuk, M. I.; Kershaw, S. V.; Kovalenko, M. V.; Rogach, A. L. Lead Halide Perovskite Nanocrystals in the Research Spotlight: Stability and Defect Tolerance. ACS Energy Lett. 2017, 2 (9), 2071-2083. https://doi.org/10.1021/acsenergylett.7b00547.

${ }^{10}$ Pan, J.; Shang, Y.; Yin, J.; De Bastiani, M.; Peng, W.; Dursun, I.; Sinatra, L.; El-Zohry, A. M.; Hedhili, M. N.; Emwas, A.-H.; et al. Bidentate Ligand-Passivated CsPbI3 Perovskite Nanocrystals for Stable Near-Unity Photoluminescence Quantum Yield and Efficient Red Light-Emitting Diodes. J. Am. Chem. Soc. 2018, 140 (2), 562-565. https://doi.org/10.1021/jacs.7b10647.

${ }^{11}$ Baek, S.; Kim, S.; Noh, J. Y.; Heo, J. H.; Im, S. H.; Hong, K.-H.; Kim, S.-W. Development of Mixed-Cation $\mathrm{Cs}{ }_{x} \mathrm{Rb}_{1-}{ }_{x} \mathrm{PbX}_{3}$ Perovskite Quantum Dots and Their Full-Color Film with High Stability and Wide Color Gamut. Adv. Opt. Mater. 2018, 6 (15), 1800295. https://doi.org/10.1002/adom.201800295.

${ }^{12}$ Saliba, M.; Matsui, T.; Domanski, K.; Seo, J.-Y.; Ummadisingu, A.; Zakeeruddin, S. M.; Correa-Baena, J.-P.; Tress, W. R.; Abate, A.; Hagfeldt, A.; et al. Incorporation of Rubidium Cations into Perovskite Solar Cells Improves Photovoltaic Performance. Science 2016, 354 (6309), 206-209. https://doi.org/10.1126/science.aah5557.

${ }^{13}$ Saliba, M.; Matsui, T.; Seo, J.-Y.; Domanski, K.; Correa-Baena, J.-P.; Nazeeruddin, M. K.; Zakeeruddin, S. M.; Tress, W.; Abate, A.; Hagfeldt, A.; et al. Cesium-Containing Triple Cation Perovskite Solar Cells: Improved Stability, Reproducibility and High Efficiency. Energy Environ. Sci. 2016, 9 (6), 1989-1997. https://doi.org/10.1039/C5EE03874J.

${ }^{14}$ Yi, C.; Luo, J.; Meloni, S.; Boziki, A.; Ashari-Astani, N.; Grätzel, C.; Zakeeruddin, S. M.; Röthlisberger, U.; Grätzel, M. Entropic Stabilization of Mixed A-Cation ABX3 Metal Halide Perovskites for High Performance Perovskite Solar Cells. Energy Environ. Sci. 2016, 9 (2), 656-662. https://doi.org/10.1039/C5EE03255E.

15 Singh, T.; Miyasaka, T. Stabilizing the Efficiency Beyond 20\% with a Mixed Cation Perovskite Solar Cell Fabricated in Ambient Air under Controlled Humidity. Adv. Energy Mater. 2018, 8 (3), 1700677. https://doi.org/10.1002/aenm.201700677.

${ }^{16}$ McMeekin, D. P.; Sadoughi, G.; Rehman, W.; Eperon, G. E.; Saliba, M.; Hörantner, M. T.; Haghighirad, A.; Sakai, N.; Korte, L.; Rech, B.; et al. A Mixed-Cation Lead Mixed-Halide Perovskite Absorber for Tandem Solar Cells. Science 2016, 351 (6269), 151-155. https://doi.org/10.1126/science.aad5845.

17 Amgar, D.; Aharon, S.; Etgar, L. Inorganic and Hybrid Organo-Metal Perovskite Nanostructures: Synthesis, Properties, and Applications. Adv. Funct. Mater. 2016, 26 (47), 8576-8593. https://doi.org/10.1002/adfm.201603752.

${ }_{18}$ Protesescu, L.; Yakunin, S.; Bodnarchuk, M. I.; Krieg, F.; Caputo, R.; Hendon, C. H.; Yang, R. X.; Walsh, A.; Kovalenko, M. V. Nanocrystals of Cesium Lead Halide Perovskites (CsPbX3 , $\mathrm{X}=\mathrm{Cl}, \mathrm{Br}$, and I): Novel Optoelectronic Materials Showing Bright Emission with Wide Color Gamut. Nano Lett. 2015, 15 (6), 3692-3696. https://doi.org/10.1021/nl5048779.

${ }^{19}$ Nedelcu, G.; Protesescu, L.; Yakunin, S.; Bodnarchuk, M. I.; Grotevent, M. J.; Kovalenko, M. V. Fast Anion-Exchange in Highly Luminescent Nanocrystals of Cesium Lead Halide Perovskites ( $\mathrm{CsPbX} 3, \mathrm{X}=\mathrm{Cl}, \mathrm{Br}, \mathrm{I})$. Nano Lett. 2015, 15 (8), 5635-5640. https://doi.org/10.1021/acs.nanolett.5b02404.

${ }^{20}$ Akkerman, Q. A.; D’Innocenzo, V.; Accornero, S.; Scarpellini, A.; Petrozza, A.; Prato, M.; Manna, L. Tuning the Optical Properties of Cesium Lead Halide Perovskite Nanocrystals by 
Anion Exchange Reactions. J. Am. Chem. Soc. 2015, 137 (32), 10276-10281. https://doi.org/10.1021/jacs.5b05602.

21 Trots, D. M.; Myagkota, S. V. High-Temperature Structural Evolution of Caesium and Rubidium Triiodoplumbates. J. Phys. Chem. Solids 2008, 69 (10), 2520-2526. https://doi.org/10.1016/J.JPCS.2008.05.007.

${ }^{22}$ Sessolo, M.; Gil-Escrig, L.; Longo, G.; Bolink, H. J. Perovskite Luminescent Materials; Springer, Cham, 2017; pp 241-268. https://doi.org/10.1007/978-3-319-59304-3_8

${ }^{23}$ Imran, M.; Ijaz, P.; Goldoni, L.; Maggioni, D.; Petralanda, U.; Prato, M.; Almeida, G.; Infante, I.; Manna, L. Simultaneous Cationic and Anionic Ligand Exchange For Colloidally Stable CsPbBr3 Nanocrystals. ACS Energy Lett. 2019, 4 (4), 819-824. https://doi.org/10.1021/acsenergylett.9b00140

${ }^{24}$ Imran, M.; Caligiuri, V.; Wang, M.; Goldoni, L.; Prato, M.; Krahne, R.; De Trizio, L.; Manna, L. Benzoyl Halides as Alternative Precursors for the Colloidal Synthesis of LeadBased Halide Perovskite Nanocrystals. J. Am. Chem. Soc. 2018, 140 (7), 2656-2664. https://doi.org/10.1021/jacs.7b13477

${ }^{25}$ Krieg, F.; Ochsenbein, S. T.; Yakunin, S.; ten Brinck, S.; Aellen, P.; Süess, A.; Clerc, B.; Guggisberg, D.; Nazarenko, O.; Shynkarenko, Y.; et al. Colloidal CsPbX3 (X = Cl, Br, I) Nanocrystals 2.0: Zwitterionic Capping Ligands for Improved Durability and Stability. ACS Energy Lett. 2018, 3 (3), 641-646. https://doi.org/10.1021/acsenergylett.8b00035

${ }^{26}$ Protesescu, L.; Yakunin, S.; Kumar, S.; Bär, J.; Bertolotti, F.; Masciocchi, N.; Guagliardi, A.; Grotevent, M.; Shorubalko, I.; Bodnarchuk, M. I.; et al. Dismantling the "Red Wall" of Colloidal Perovskites: Highly Luminescent Formamidinium and Formamidinium-Cesium Lead Iodide Nanocrystals. ACS Nano 2017, 11 (3), 3119-3134. https://doi.org/10.1021/acsnano.7b00116

${ }^{27}$ Amgar, D.; Binyamin, T.; Uvarov, V.; Etgar, L. Near Ultra-Violet to Mid-Visible Band Gap Tuning of Mixed Cation $\mathrm{Rb}_{\mathrm{x}} \mathrm{Cs}_{1-\mathrm{x}} \mathrm{PbX}_{3}(\mathrm{X}=\mathrm{Cl}$ or $\mathrm{Br})$ Perovskite Nanoparticles. Nanoscale 2018, 10 (13), 6060-6068. https://doi.org/10.1039/C7NR09607K

${ }^{28}$ Udayabhaskararao, T.; Kazes, M.; Houben, L.; Lin, H.; Oron, D. Nucleation, Growth, and Structural Transformations of Perovskite Nanocrystals. Chem. Mater. 2017, 29 (3), 13021308. https://doi.org/10.1021/acs.chemmater.6b04841

${ }^{29}$ Hohenberg, P.; Kohn, W. Inhomogeneous Electron Gas. Phys. Rev. 1964, 136 (3B), B864B871. https://doi.org/10.1103/PhysRev.136.B864.

${ }^{30}$ Kohn, W.; Sham, L. J. Self-Consistent Equations Including Exchange and Correlation Effects. Phys. Rev. 1965, 140 (4A), A1133-A1138.

https://doi.org/10.1103/PhysRev.140.A1133.

${ }^{31}$ Soler, J. M.; Artacho, E.; Gale, J. D.; García, A.; Junquera, J.; Ordejón, P.; Sánchez-Portal, D. The SIESTA Method for Ab Initio Order- $N$ Materials Simulation. Journal of Physics: Condensed Matter 2002, 14 (11), 2745-2779. https://doi.org/10.1088/0953-8984/14/11/302.

${ }^{32}$ Artacho, E.; Anglada, E.; Diéguez, O.; Gale, J. D.; García, A.; Junquera, J.; Martin, R. M.; Ordejón, P.; Pruneda, J. M.; Sánchez-Portal, D.; et al. The SIESTA Method; Developments and Applicability. Journal of Physics: Condensed Matter 2008, 20 (6), 064208. https://doi.org/10.1088/0953-8984/20/6/064208.

33 Amgar, D.; Wierzbowska, M.; Uvarov, V.; Gutkin, V.; Etgar, L. Novel Rubidium Lead Chloride Nanocrystals: Synthesis and Characterization. Nano Future 2017, 1 (2), 021002. https://doi.org/10.1088/2399-1984/aa8761

${ }^{34}$ H. Monzel, M. Schramm, K. Towe, H. P. Beck. Zur Neuuntersuchung des Phasendiagramms $\mathrm{RbCl} / \mathrm{PbCl}_{2}$, Z. Anorg. Allg. Chem. 2000, 626, 408. 